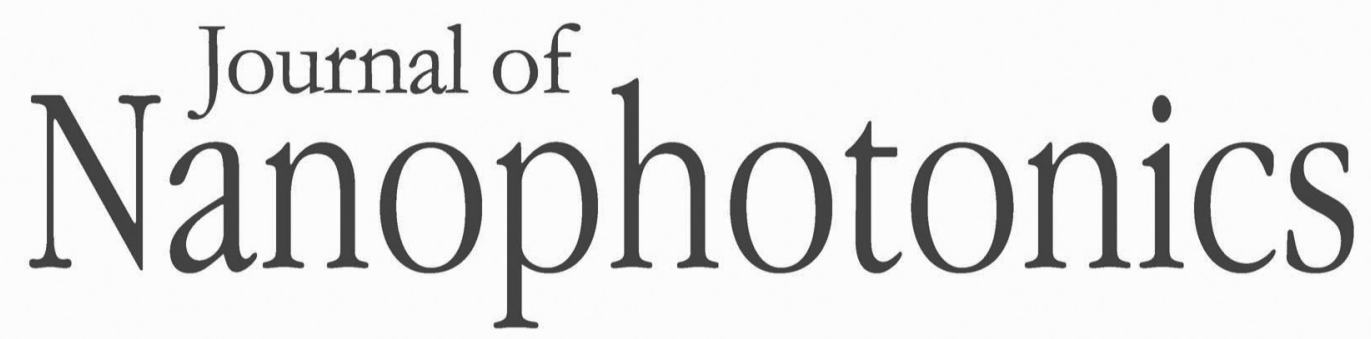

SPIEDigitalLibrary.org/jnp

\title{
Silicon Photonics for Telecommunications and Biomedicine
}

Samia A. Suliman 


\section{BOOK REVIEW}

\section{Silicon Photonics for Telecommunications and Biomedicine}

Sasan Fathpour and Sasan Fathpour, Eds., xiii+ 447 pages, ISBN 978-1-4398-0637-1, CRC Press, Boca Raton, Florida (2012), US \$86.11, hardcover.

Reviewed by Samia A. Suliman, Pennsylvania State University, Department of Engineering Science and Mechanics, University Park, Pennsylvania 16802

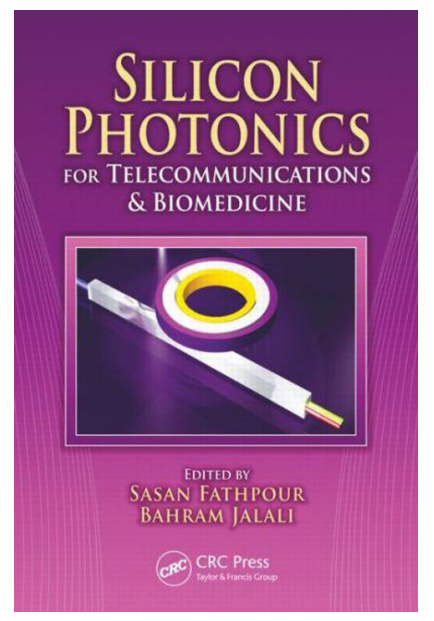

The silicon metal-oxide-semiconductor field-effect transistor (MOSFET) is the basic unit of digital technology that has altered the shape, form, pace, and the very nature of human interactions on planet Earth. Silicon is one of the most studied materials in the history of man, the other two being steel and cement. The study of silicon is unique and today $90 \%$ of photovoltaic cells are silicon-based. In addition to its excellent electronic semiconducting properties, silicon's mechanical properties render it a basic element for micro-electro-mechanical systems (MEMS).

The silicon MOSFET's successful and continuous miniaturization in the last six decades is today confronted with numerous challenges that can be summarized into three facets: the quantum-mechanical limits imposed on the principle of device operation, power dissipation, and the exponentially increasing cost of setting up new semiconductor fabrication plants. The last of the three is affectionately termed Moore's second law.

Industry focus is now shifting from the vertical pursuit of further miniaturization to the horizontal enhancement of functionality of the technology in other arenas. For example, a field of application that has benefited from this aggressive lateral shift of industry is power electronics.

Twenty percent of the global electricity consumption is in lighting, while $12 \%$ of the energy is consumed worldwide in handheld electronics. The recent focus on silicon photonics and its applications promises tremendous possibilities for the solution of the impending energy crisis.

In their comprehensive compilation of research activities in utilizing silicon in a new arena, Sasan Fathpour and Bahram Jalali have opened my eyes to silicon-photonics applications in telecommunications. The conjunction of solid-state physics, electrical engineering, and material science has given birth to spectacular digital technology and taught humanity a valuable lesson of the tremendous value of multidisciplinary work. It astonished me that the microelectronically rudimentary structure of intrinsic silicon coupled with a metal can be utilized as a basic unit of silicon plasmonics, namely, a waveguide. In line with bandgap engineering we now have density-of-carriers engineering.

The advantage of integrating silicon photonics with electronics is the low cost associated with complex optics functionality. Optical pathways consume less energy in comparison to metallic interconnects. As a result, photonics provides a solution to the power dissipation issue while performing inter- and intrachip information transfer at the speed of light.

The milestones for the potential of silicon-photonics integration are the realization of micrometer-scale waveguides, modulation of the free-carrier density, strained germanium on silicon layers for infrared detectors, and commercial emergence of silicon-on-insulator as a

0091-3286/2012/\$25.00 (C) 2012 SPIE 
platform for optical confinement layers, coupled with inherent advantages of silicon as an element.

The first chapter of the book deals with the evolution of silicon photonics since its inception in the mid-1980s. During the 1990s the focus was on the waveguides. Silicon optical modulators as transceivers rely on carrier injection, accumulation, and depletion for changing the refractive index in a waveguide as the basic telecommunication component. The research trends are for smaller waveguides to reduce power consumption and real estate. In addition, the chapter discusses key technological challenges: light sources, power consumption, couplers, packaging, and fiber alignments, not to mention size differences in photonics and electronics relevant to plasmonic waveguides.

Silicon plasmonics as the newcomer to plasmonic literature is the subject of the second chapter. Silicon-plasmonic waveguides have been built and tested. The structure is simple consisting of layers of conductors and dielectrics. The possibility of integrating plasmonics with electronics and with photonics for potential plasma-opto-electronic integrated circuits (POEIC) is discussed.

The importance of stress engineering in altering the linear and nonlinear optical properties of silicon is discussed in Chap. 3. In particular, dynamic tuning of a silicon waveguide's birefringence is achieved by integrating a piezoelectic transducer (PZT). The second case of stress engineering studied is the periodically poled silicon (PePSi) photonic device.

Chapter 4 covers the physics of the nonlinear optical effect of two-photon absorption (TPA), which is particularly strong in semiconductors. TPA and TPA-induced free-carrier absorption (FCA) and their roles in pulse-shaping mechanisms are discussed. FCA is reduced either by ion implantation or by using the $p-i-n$ structure to reduce carrier lifetime.

The theory of silicon Raman amplifiers and lasers is the topic of Chap. 5 coupled with an extensive list of references. Chapter 6 is focused on the biomedical applications of silicon photonics. The role of silicon as a photonic material in the technologically important midinfrared regime and its interaction with biological materials is discussed in Chap. 7. The growth of III-V material on silicon is covered in Chap. 8. Chapter 9 discusses the hybrid silicon group III-V lasing applications. The 3-D integration of CMOS with photonics is the subject of Chap. 10. The subject of nonlinear photonics and energy harvesting is tackled in Chap. 11. The book concludes with Chap. 12 on computer-aided designs for CMOS photonics.

With the amalgamation of the state of the art in silicon photonics work and its concise presentation coupled with the provision of an extensive list of references, I highly recommend the book for all those embarking on graduate studies in the field and its related areas; in addition I highly recommend it for all $\mathrm{R} \& \mathrm{D}$ groups in the semiconductor industry. 